http://jmscr.igmpublication.org/home/ ISSN (e)-2347-176x ISSN (p) 2455-0450 crossref DOI: https://dx.doi.org/10.18535/jmscr/v7i8.28

\title{
Clinical study to compare the short term outcomes in six months follow up period with Respect to open mesh and non mesh repair based on randomization
}

\author{
Authors \\ Dr Vasanth Dunna ${ }^{1}$, Dr Uday Kiran Mokati ${ }^{2 *}$, Dr Kamala Priya Tata ${ }^{3}$, \\ Dr Narendra Babu Bathina ${ }^{4}$, Dr Mohammad Nowsheen ${ }^{5}$, Dr D V Lavanya Ambati ${ }^{6}$, \\ Dr Lakshmi Sri Durga Mallela ${ }^{7}$, Sree Siddi Tilak Pasupuleti ${ }^{8}$ \\ ${ }^{1,2}$ Asst. Professor, Dept of General Surgery, Konaseema Institute of Medical Sciences and Research Foundation, \\ Amalapuram, Andhra Pradesh, India- 533201 \\ ${ }^{3,7}$ First Year Post Graduate, Dept of General Surgery, Konaseema Institute of Medical Sciences and Research \\ Foundation, Amalapuram, Andhra Pradesh, India- 533201 \\ ${ }^{4}$ Final Year Post Graduate, Dept of General Surgery, Konaseema Institute of Medical Sciences and Research \\ Foundation, Amalapuram, Andhra Pradesh, India- 533201 \\ ${ }^{5,6}$ Second Year Post Graduate, Dept of General Surgery, Konaseema Institute of Medical Sciences and Research \\ Foundation, Amalapuram, Andhra Pradesh, India- 533201 \\ ${ }^{8}$ Final Year Part 2 MBBS, Konaseema Institute of Medical Sciences and Research Foundation, Amalapuram, Andhra \\ Pradesh, India- 533201 \\ *Corresponding Author \\ Dr Uday Kiran Mokati
}

Asst. Professor, Dept of General Surgery, Konaseema Institute of Medical Sciences and Research Foundation, Amalapuram, Andhra Pradesh, India- 533201

\section{Abstract}

Background: Hernia are among the oldest surgical challenges and are currently the most commonly performed general surgical operations.

Many of the newer techniques, have demonstrated that when performed well, the recurrence rate should be relatively low.

Objective: The objective is to know the risk factors, clinical presentations, and various methods of surgical treatment, and also the comparative study between open mesh and non mesh repair of inguinal hernia in six month duration of follow up.

Method: A randomized comparative study of Open Mesh and Non-mesh repair was done for the 135 patients presenting with inguinal hernia, admitted in our hospital were studied from January 2017 to June 2018.

Results: Out of 135 patients who underwent inguinal hernia surgery, majority were men in their fourth and fifth decade. Most of the patients opted for Non-mesh type of repair.

Randomized prospective study was done to compare Non-mesh and mesh repair of inguinal hernia. Patients were followed for six months, except for 3recurrences in Non-mesh group, there were no other significant difference noted.

Conclusion: After Randomized study, it wasn't possible to identify clearly the benefits of mesh repair in respect to short term outcomes.

Though it is advised, particularly in training institution, to use mesh because Recurrence rate is low. The present study results indicate that this is not the case of early recurrence, which represent technical failure. And also, studies says that long term follow-up is required for full determination of recurrence rate.

Keywords: Inguinal Hernia, Direct, Indirect. Mesh Repair, Non Mesh Repair. 


\section{JMSCR Vol||07||Issue||08||Page 164-173||August}

\section{Introduction}

Hernias may be generally defined as a protrusion of abdominal viscera outside the abdominal cavity through a natural or acquired defect. The Latin word hernia means rupture or tear. Hernia is not a disease of modern society; its occurrence was noted during early $16^{\text {th }}$ century $\mathrm{BC}$ and was recognized as a surgical disease by Praxagoras of Kos because of its demand for some sort of lifesaving treatment ${ }^{[1]}$

"A protrusion of any viscus from its proper cavity is denominated a hernia. The protruded parts are generally contained in a bag by a membrane with which the cavity is naturally invested" - Sir Astley Cooper 1804.

Inguinal hernias are among the most common problems encountered by the Surgeon. Seventy five percent of all abdominal wall hernias occur in the groin. Indirect hernia out number direct hernias by about 2:1 with femoral hernia making up a very small proportion. Sir Astley Cooper stated "No disease of the human body, belonging to the province of surgeon, requires in its treatment a better combination of accurate anatomical knowledge with surgical skill than hernia in all its varieties". A thorough knowledge is a must for any surgeon.

Though inguinal hernia is a very common ailment amongst surgical patients, irreducibility, obstruction and strangulation are its commonest complication encountered in surgical practice as acute emergency. The identification of risk factors like age, sex, type of hernia, presenting symptoms and associated preexisting medical illness helps give priority in admission to such patients. The delay in surgery in a case of complicated hernia increases the morbidity and mortality in such patients.

So this study is being done to evaluate the risk factors, clinical presentations, complications, management protocol and comparison between open mesh and non-mesh repair short term outcomes in six months follow up period.

\section{Results}

A total of 135 patients with inguinal hernia admitted, investigated, operated and followed up are studied. The results are as follows:

Patient's demography

Graph 1: Age Distribution

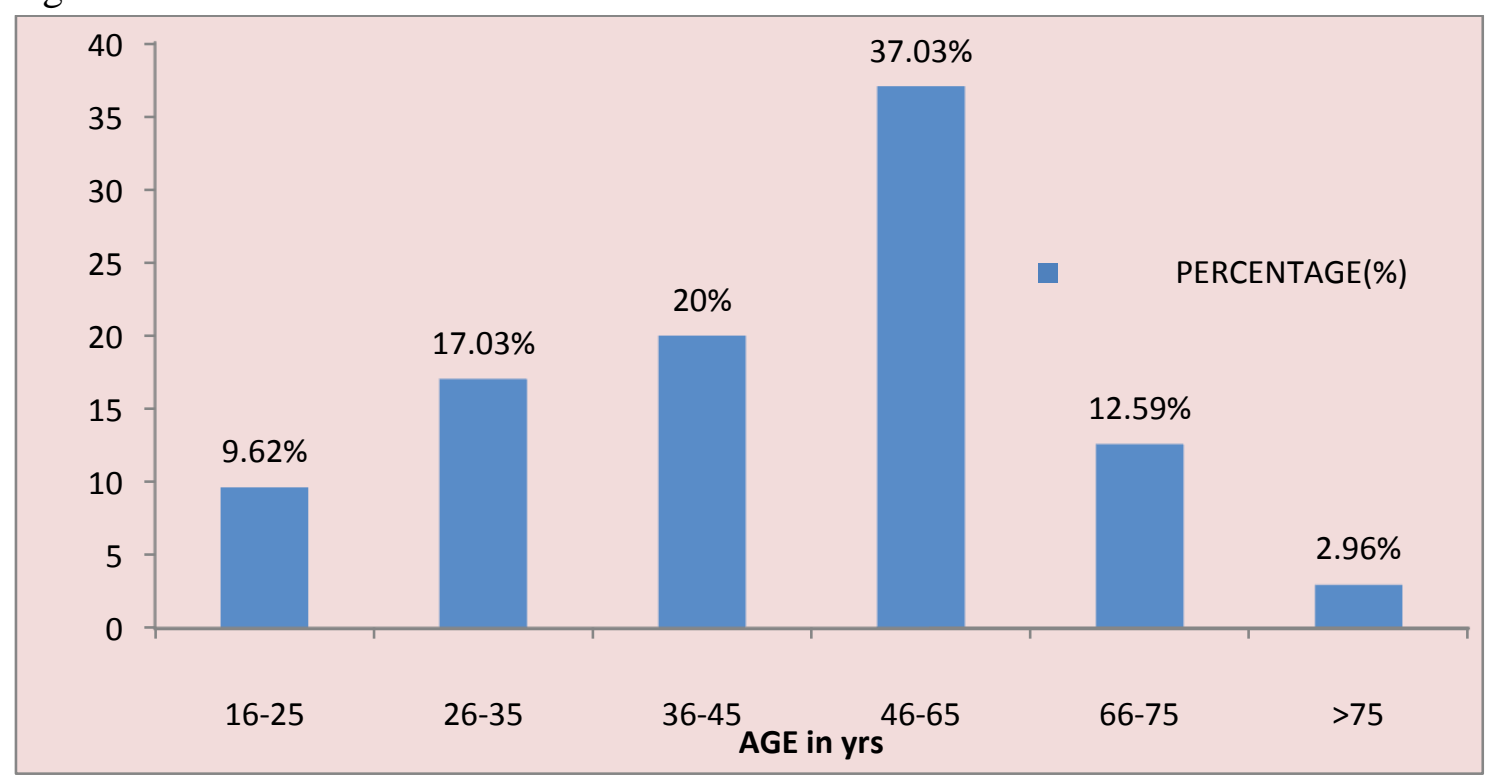

The youngest patient included in this series was $37 \%$ of patients were in $46-65$ year age group. 18 Years and the eldest was 82 Years old. Almost 


\section{Sex Distribution}

Graph 2: Sex Distribution

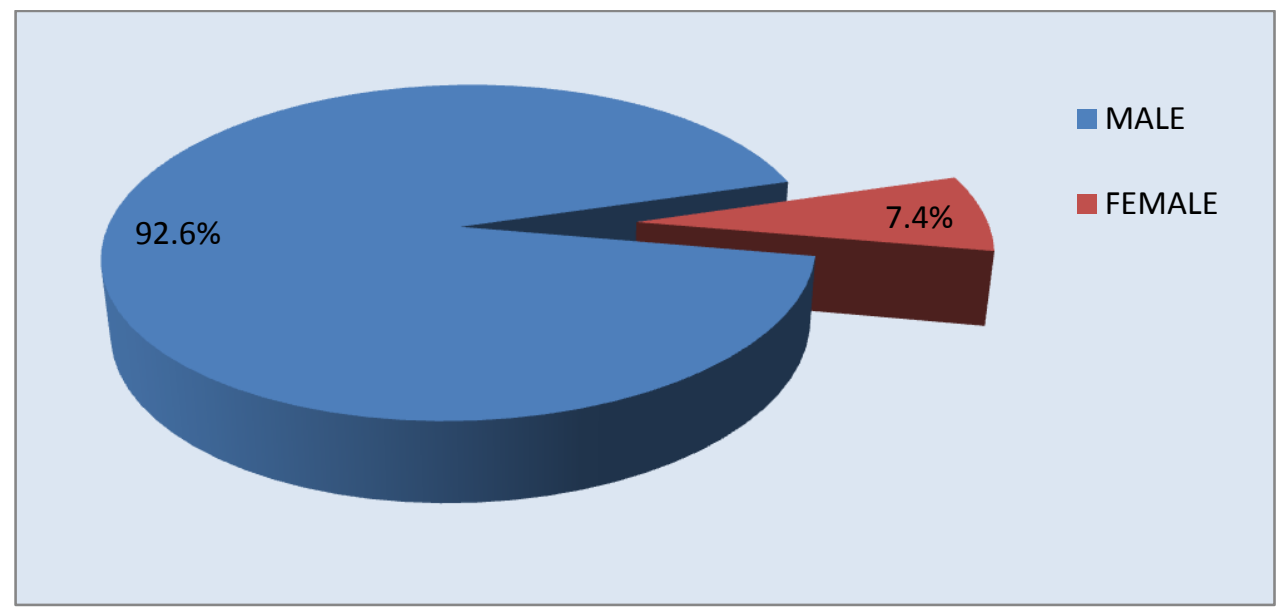

Only $7 \%$ of patients in this study were females, as compared to males who made $93 \%$ of total cases.

\section{Clinical Presentation}

Graph 3: Clinical Presentation

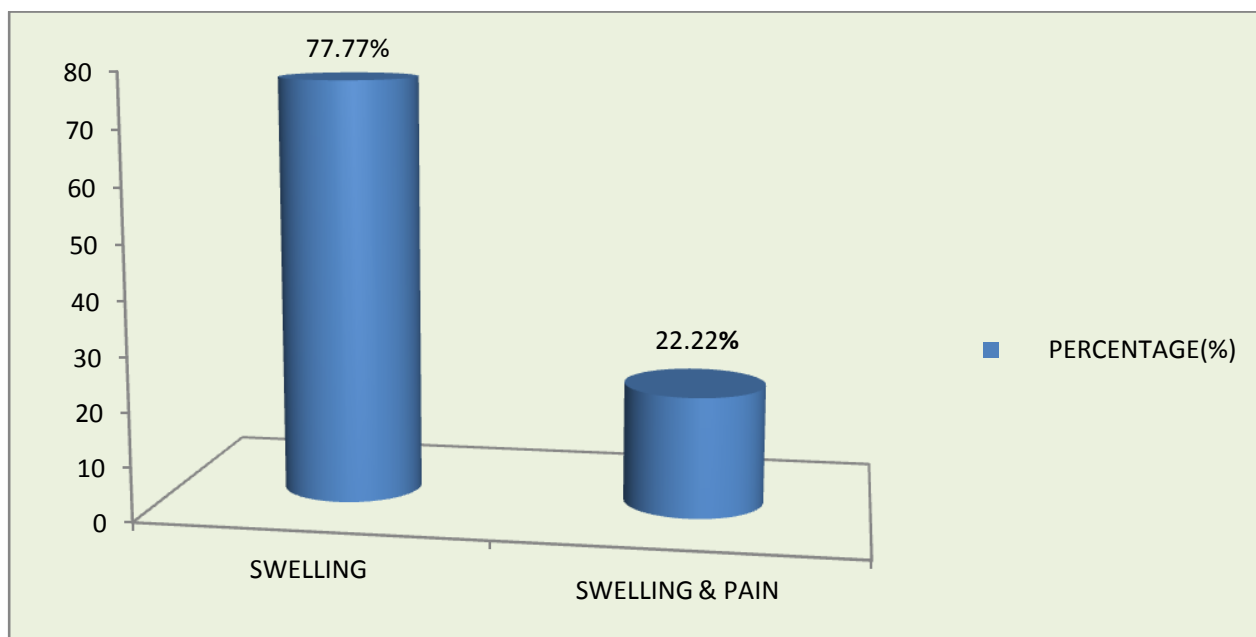

Although all the patients in the study presented with swelling in the inguinal region, associated pain was present in $22 \%$ of patients.

\section{Duration of Illness}

Graph 4: Duration of Illness

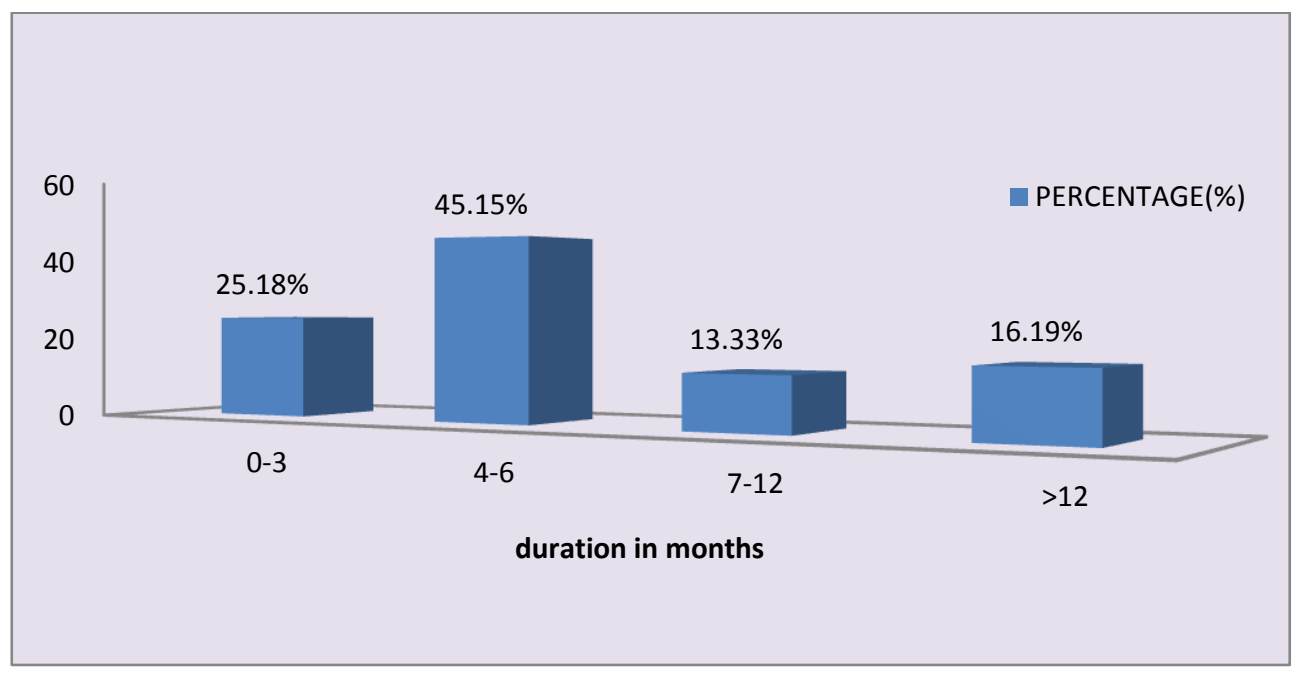


Among the patients studied, $25 \%$ came to hospital by 3 months after appearance of swelling \& $84 \%$ by 1 year $\& 16 \%$ of patients took more than 1 year.
Majority of the cases of (about 45\%) presented with 4-6 months duration of symptoms.

\section{Side of Inguinal Hernia}

Graph 5: Side of Inguinal Hernia

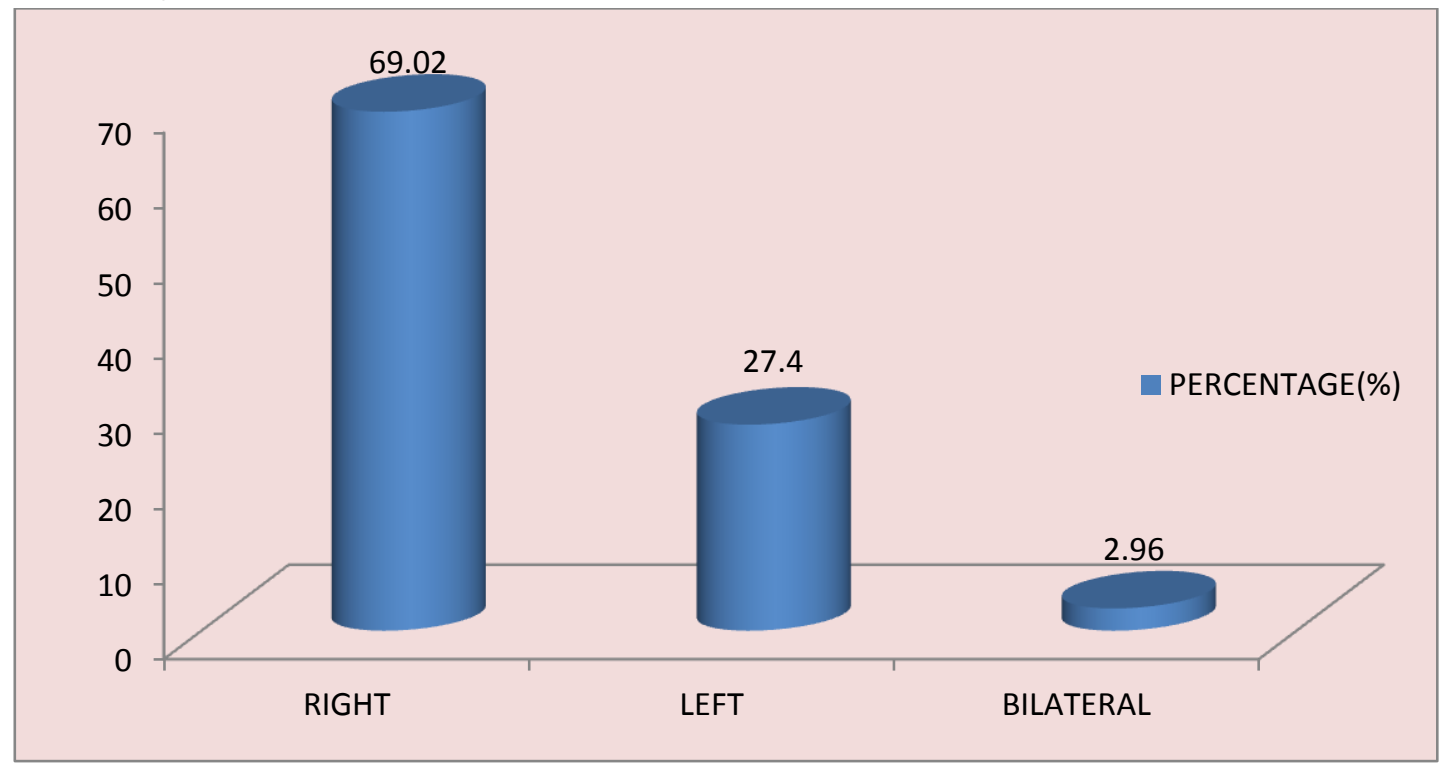

The study showed that incidence of hernia is more on right side. Left sided hernia comprised of about $27 \%$ of cases, whereas $3 \%$ of cases were bilateral.

\section{Recurrent Inguinal Hernia}

Graph 6: Recurrent Hernia

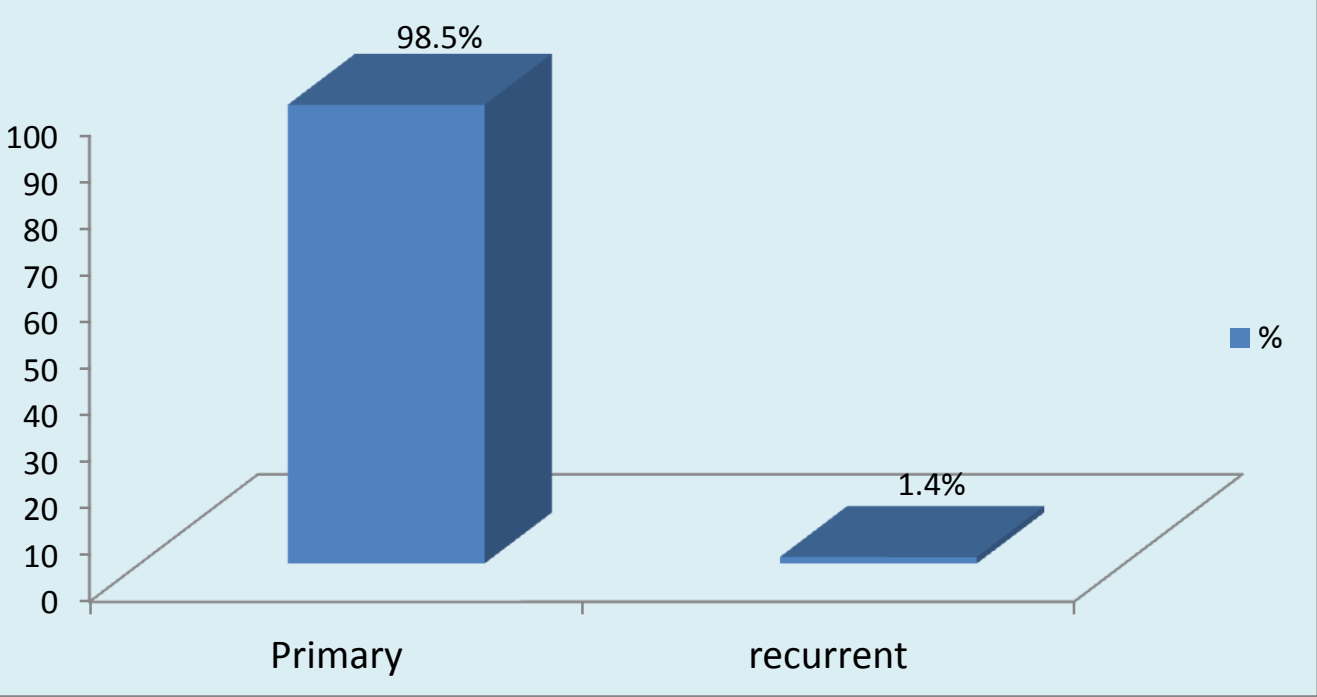

The study showed only $<2 \%$ of recurrent hernia cases among 135 cases collected. Both the cases had undergone non-mesh type of repair (darning). At surgery, in one case it was found that hernia was from the deep ring, due to inadequate closure of deep ring. In other case of recurrent hernia, it was found that there was breach in the reflected part of inguinal ligament through which posterior wall was repair. 
Type of Inguinal Hernia

Graph 7: Type of Inguinal Hernia

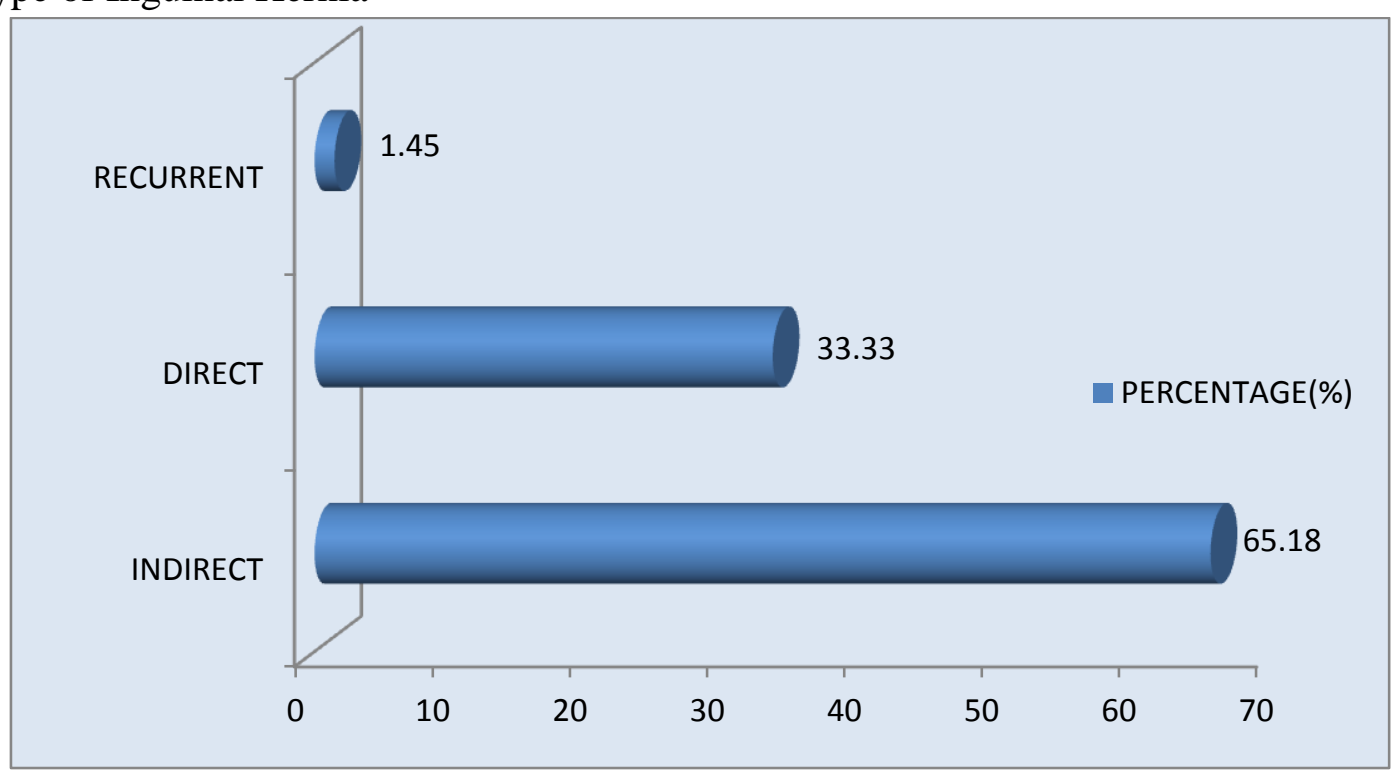

In the 135 cases studied, majority of the cases were of indirect variety (65\%).

\section{Comparison of Study Groups}

A total of 135 patients of inguinal hernia were randomized. Among them 75 belong to mesh group \& 60 belong to non-mesh group.

\section{Duration of Surgery}

Graph 8: Duration of Surgery

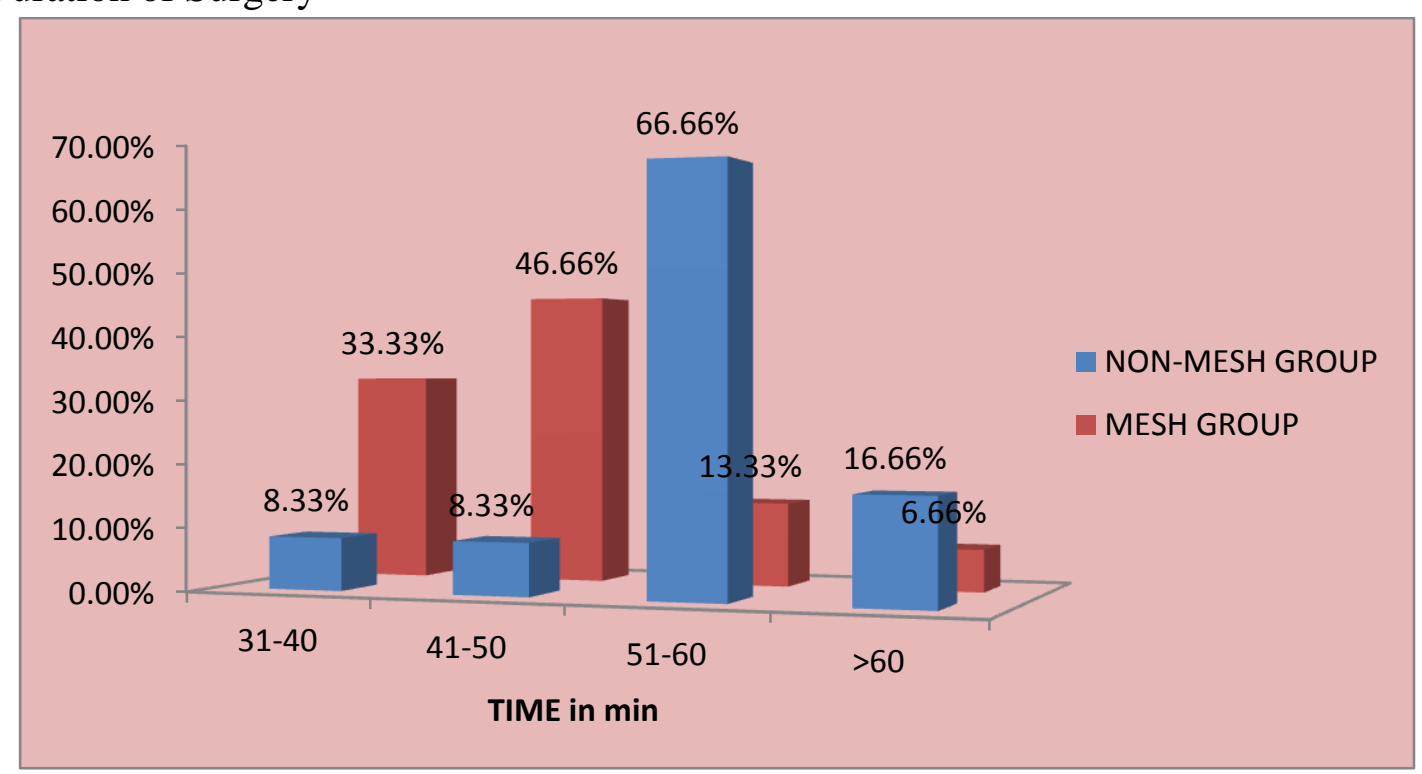

Mean duration of surgery in mesh group is 46.33 minutes.

Mean duration of surgery in non-mesh group is 54.33 minutes. 


\section{Hospital Stay}

Graph 9: Hospital Stay

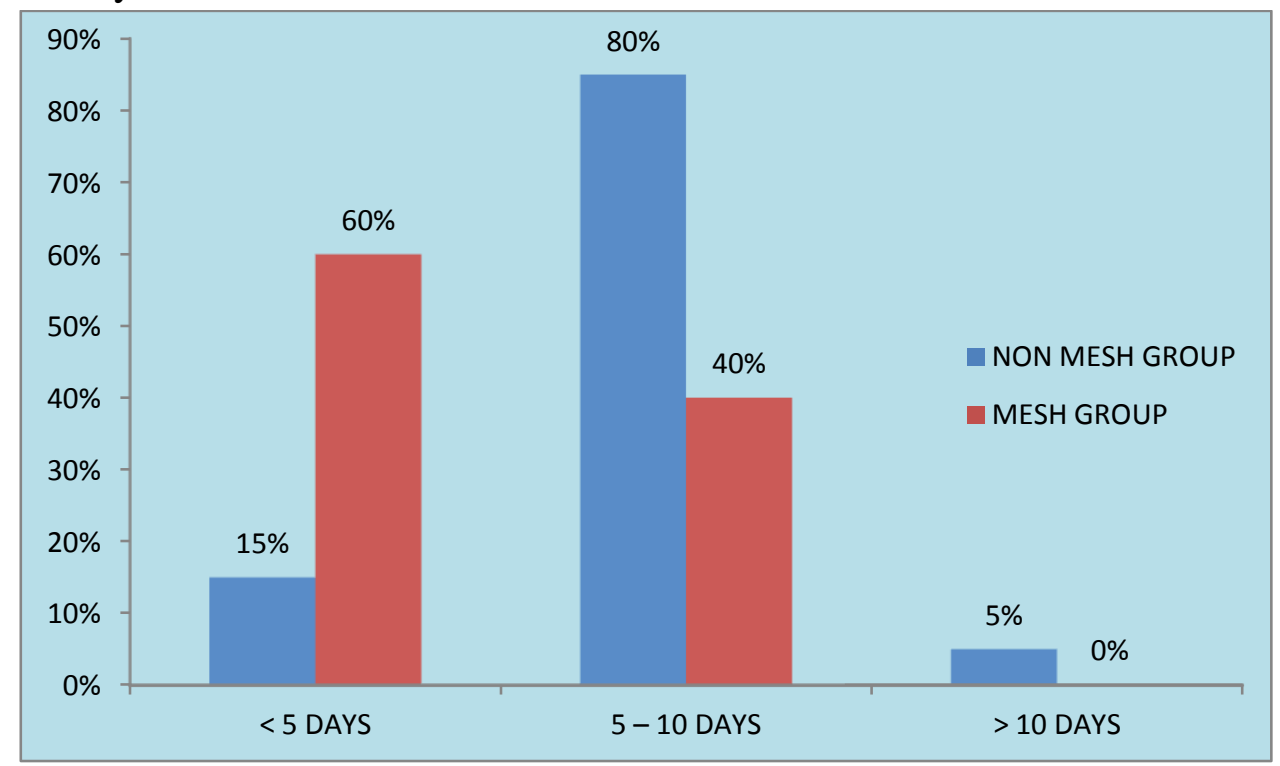

Mean hospital stay in mesh group is 5.6 days.

Mean hospital stay in non mesh group is 6.53 days.

\section{Complications in Follow-up Period}

Graph 10: Complications in Follow up

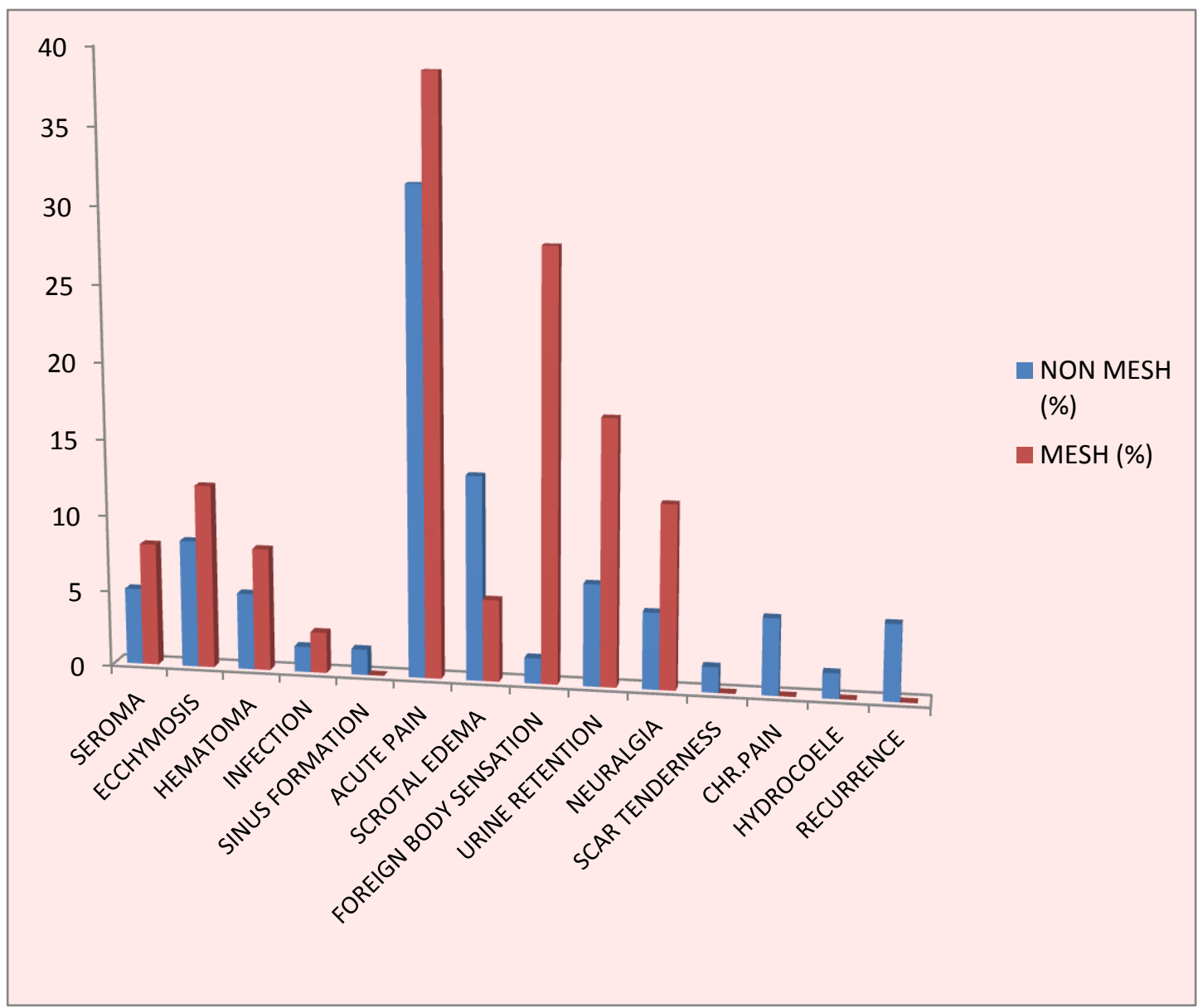

The above table shows the cumulative complication in mesh and non-mesh group in one year of follow-up period. 


\section{Clinical Presentation of Inguinal Hernia Symptoms}

1. Pain: The commonest symptom of hernia is discomfort or pain. Patient complains of a dragging or aching type of pain in the groin which gets as the day passes. This is often noticed when there is a tendency to hernia. So pain may appear long before the lump is noticed. Pain continues so long as the hernia is progressing, but ceases when it is fully formed. In well formed hernia, there is a sense of heaviness or weight. Pull on the mesentry may cause pain in the epigastric region. The patient may complain pain all over the abdomen due to drag on the mesentry and omentum.

2. Lump: Many hernias may cause no pain and patient has noticed only a swelling inthe groin.

3. Systemic symptoms: If the hernia is obstructing the lumen of the bowel, cardinal symptoms of intestinal obstruction will appear.

4. Other complaints: Precipitating factors like, persistent cough, chronic bronchitis, constipation, dysuria due to benign enlargement of prostate, or stricture urethra.

5. Past history: During appendicectomy division of sub costal or ilio inguinal nervemay lead to weakness of abdominal muscles at the inguinal region and may cause subsequent direct inguinal hernia. Sometimes hernias are thought to be the result of a single event for e.g. lifting a heavy object, but in fact repetitive mechanical strain is possibly the damaging factor ${ }^{[2,4]}$. Inside the abdominal cavity a continuous positive pressure of $2-20 \mathrm{~mm} \mathrm{Hg}$ is maintained. This pressure can increase to values as high as $150 \mathrm{~mm} \mathrm{Hg}$ during coughing and vomiting ${ }^{[3]}$. Recent day's research targets connective tissue disorders in the process of hernia development due to its primary role in the linking of abdominal organs ${ }^{[5]}$.

\section{Signs}

Two classical signs of uncomplicated hernias are impulse on coughing and reducibility.

-To get above the swelling differentiates a scrotal from an ingino-scrotal swelling.

-If the inguinal hernia contains omentum the swelling feels doughy and granular. If it contains intestine, it feels elastic. A strangulated hernia feels tense and tender.

-An expansile impulse on coughing in the hernia will be appreciated when the patient is asked to cough. Impulse on coughing will be absent in case of strangulated, irreducible, and obstructed hernia. -Ring occlusion test: After reducing the hernia the deep inguinal ring is occluded, i.e., half an inch above mid inguinal point . On asking the patient to cough, a direct hernia will show a bulge but an indirect hernia will not find access, so no bulge.

\section{Investigations}

Routine laboratory investigations like Hemoglobin\%, urine routine, blood urea, serum creatinine will aid in the search of normal parameters before taking the patient for Surgery. Roentgen graphic examination of the abdomen may reveal the patterns characteristic of intestinal obstruction with air and fluid filled loops of intestine on Plain x-ray erect abdomen as in complicated presentations of inguinal hernias.

Ultrasound of the abdomen to know the obstructive urinary outflow diseases and Chest $\mathrm{x}$ ray to find pulmonary pathology

\section{Surgical Management of Inguinal Hernia}

Surgical repair of groin hernia can be quite simple, but in some cases, the distortion and attenuation of normal anatomy provide an extreme technical challenge. Inguinal hernias should be surgically repaired after diagnosis by physical examination. Until 1958, the treatment for abdominal wall hernias are suture based and the major problem faced by the then surgeons were the increased recurrence of hernia ${ }^{[6]}$. A terminally ill, immune suppressed, extremely old, or unacceptable surgical risk patient may fall into the category of those who should be followed without operative correction. The natural history of groin hernia is one of the progressive enlargements and weakening, whiles the potential for Incarceration and obstruction of the intestine and subsequent of the vascular supply to the bowel (strangulation), 
leading to intestinal infarction. Hernias do not resolve spontaneously or improve with time. Wearing a truss does not cure a hernia, but in some patients it provides a sense of support. The extended use of a Truss can lead to increased scar formation in the inguinal region. Repair of the groin hernia can be planned electively unless incarcerated or strangulation is present.

Open hernia is begun with a straight or curvilinear incision approximately two finger breadths above inguinal ligament. Dissection is carried through the subcutaneous tissues, and the external oblique fascia is incised. Injury to the ilio-inguinal and ilio-hypogastric nerves, which provide cutaneous innervation of the skin of the lower abdomen, penis and scrotum, is avoided. The spermatic cord is mobilized by placing a finger around a cord structure at the level of pubic tubercle. Mobilization of the spermatic cord lateral to the pubic tubercle can cause improper identification of tissue planes and result in disruption of the floor of inguinal canal, with damage to the transversalis fascia. The cremaster muscle fibers of the mobilized cord are divided and separated from the underlying cord structures. The hernial sac is carefully dissected free from the adjacent cord structures and cleared to the level of internal inguinal ring. The hernia sac is opened and examined for visceral contents. The neck of the sac is suture ligated at the level of internal ring, and excess sac is excised. The options for the construction of inguinal floor are numerous and several of the more popular approaches are described. Various conventional methods like Bassini's and Shouldice repair using suture material are in practice ${ }^{[7]}$.

Bassini Repair

McVay (Cooper ligament) repair

Shouldice (Canadian) repair:

Lichtenstein (Tension free repair)

Laparoscopic hernia repair

Bassini's approach was to perform "a radical cure of inguinal hernia," and his operation epitomized the essential steps of an ideal tissue repair ${ }^{[8]}$

\section{Surgical Complications \\ Intra- operative}

a) Hemorrhage

b) Severance of nerves: The ilio inguinal and ilio hypogastric nerves and both the genital and femoral branches of genitofemoral nerves are vulnerable to injury.

c) Severance of testicular blood supply: may lead to atrophy of testis.

d) Severance of Vas Deference: If cut accidentally, anastomized with fine sutures.

e) Damage to the intestine: Usually occurs during ligation of sac.

f) Injury to urinary bladder

\section{Postoperative}

a) General Atelectasis and pneumonitis are more frequent, followed by thrombophlebitis and urinary retention.

b) Scrotal ecchymosis: found first or second postoperative day due to Dissection and blood in scrotum. It resolves spontaneously within first few weeks post operatively.

c) Swollen testis: is due to disruption of lymphatics. It is decreased by scrotal support.

d) Testicular atrophy: is prone after repair of an indirect complete scrotal hernia.

e) Wound infection.

f) Missed hernia: During repair of direct hernia, a small indirect hernia may be missed.

g) Recurrence: Mostly due to faulty surgical technique.

h) Shrinkage of mesh : This response can vary depending on its density, filament size, pore size, architecture, and the individual response of each carrier $^{[9]}$ The clinical consequences of an intense biological response can be chronic pain, intestinal adhesions and discomfort ${ }^{[10,11]}$.

i) Adhesions: adhesion of intestine with hernia meshes usually occurs within a week of the initial surgery ${ }^{[12]}$. Thereafter, a layer of peritoneal cells coat the mesh and prevent the further risk of adhesion formation ${ }^{[13]}$. 
From this finding, it is found that synthetic meshes only need a temporary adhesion barrier, hence the use of absorbable polymer coatings.

\section{Prosthetic Implants for Hernia Repair}

Usher in 1958 first used polypropylene mesh for the repair of abdominal wall defects. Lichtenstein presented his open mesh repair technique for inguinal hernia in 1986 and some years later mesh insertion using an endoscopy approach became established. Mesh insertion reduced operation time and hospital stay, and became standard practice worldwide.

With improper scar fixation of the mesh, recurrence develops at the mesh borders, particularly if the overlap is inadequate. Biologic meshes, on the other hand may gain importance in future as they have been proposed to be advantageous in contaminated areas but they are extremely expensive, not widely available and studies supporting use of biologic meshes is limited which needs further in depth analysis ${ }^{[14,15]}$

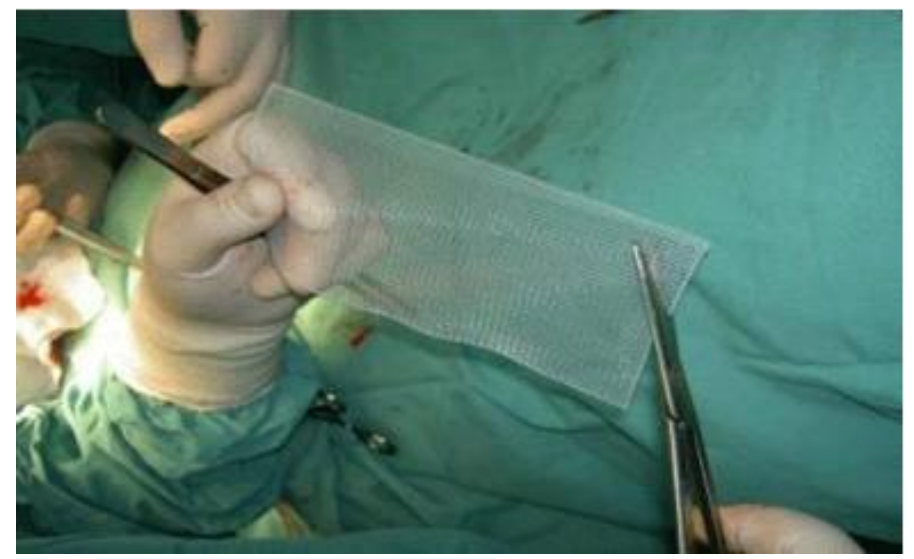

Figure 1: Polypropylene Mesh

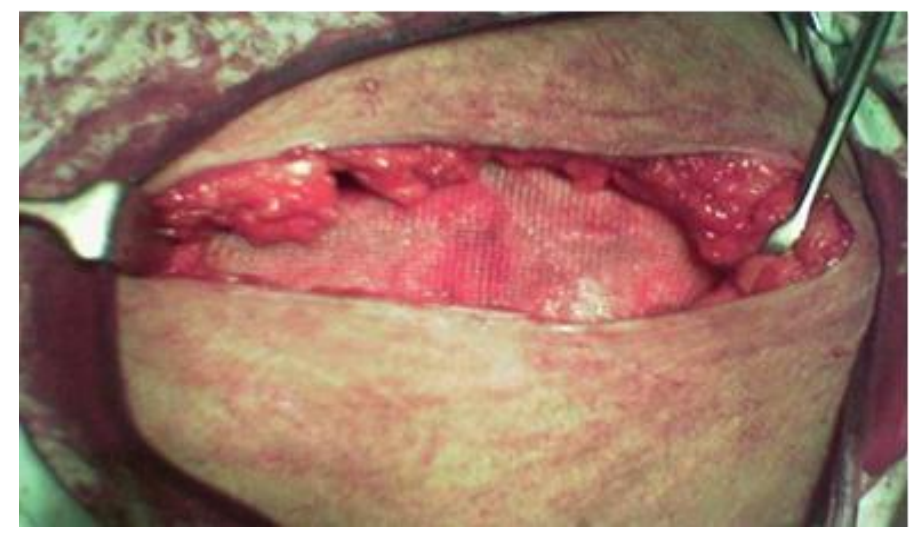

Figure 2: Open Mesh Repair

\section{Conclusion}

Perhaps the general agreement in hernia surgery that 'mesh is better than no mesh' should be followed by 'less is more' with respect to the structure of mesh.

\section{Acknowledgements}

The completion of this research paper could not have been possible without the contribution and support of so many people whose names not all may be enumerated. Firstly, we would thank the Great almighty for his precious grace on us without which this could not be possible. Secondly, we would thank the Management, Dean, Principal, Vice principal, Head of Department (HOD), and all the assisting staff of our hospital who made this article noteworthy. We are very much grateful to the participants of this study without whom this research could not be carried out.

\section{References}

1. Papavramidou NS, Christopoulou-Aletras H. Treatment of "hernia" in the writings of Celsus (First Century AD). World J Surg. 2005; 29: 1343-1347.

2. Killeen KL, Girard S, DeMeo JH, Shanmuganathan K, Mirvis SE. Using CT to diagnose traumatic lumbar hernia. AJR Am J Roentgenol. 2000; 174: 1413-1415.

3. Yu CY, Lin CC, Yu JC, Liu CH, Shyu RY, Chen CY. Strangulated transmesosigmoid hernia: CT diagnosis. Abdom Imaging.2004; 29: 158-160.

4. Kang SK, Burnett CA, Freund E, Sestito J. Hernia: is it a work-related condition? Am J Ind Med. 1999; 36(6): 638-644.

5. Sorenson LT, Jorgenson LN, Gottrup F. Biochemical aspects of abdominal wall hernia formation and recurrence. In: Nyhus and Condon's hernia, Fitzgibbons RJ Jr, Greenburg AG. (eds), Lippincott Williams \& Wilkins, Philadelphia: 2002: 9-16. [Google Scholar] 
6. Anthony $\mathrm{T}$, Bergen PC, Kim LT, Henderson M, Fahey $\mathrm{T}$, Rege RV, et al Factors affecting recurrence following incisional herniorrhaphy. World J Surg. 2000; 24: 95-101. [PubMed] [Google Scholar]

7. Malik A M, Khan A, Jawaid A, Laghari A A, Talpur K A H. A Comparative analysis between Non Mesh (Bassini's) and Mesh (Lichtenstein) Repair of Primary Inguinal Hernia. J Ayub Med Coll Abbottabad 2009;21(1)

8. Read RC. The centenary of Bassini's contribution to inguinal herniorrhaphy. Am J Surg. 1987; 153:324-326

9. Leber GE, Garb JL, Alexander AI, Reed WP. Long-term complications associated with prosthetic repair of incisional hernias. Arch Surg. 1998; 133: 378-382

10. Cobb WS, Kercher KW, Heniford BT. The argument for lightweight polypropylene mesh in hernia repair. SurgInnov. 2005; 12: 63-6911. Robinson TN CJ, Schoen J, Walsh MD. Surgical Endoscopy.2005; 19: 1556-1560.

11. Klinge U, Prescher A, Klosterhalfen B, Schumpelick V. Development and pathophysiology of abdominal wall defects. Chirurg. 1997; 68 (4):293-303.

12. Todd Vassalli J. Development of electrospun synthetic bioabsorbable fibers for a novel bionanocomposite hernia repair material. (Master of Degree thesis) Faculty of the Graduate School, University of Missouri , 2008.

13. Franklin ME, Gonzalez JJ, Glass JL. Use of porcine small intestinal submucosa as a prosthetic device for laparoscopic repair of hernias in contaminated fields: 2-year follow-up. Hernia. 2004;8:186-189.
14. Ansaloni L, Catena F, Coccolini F, Gazzotti F, D’Alessandro L, Pinna AD. Inguinal hernia repair with porcine small intestine submucosa: 3-year follow-up results of a randomized controlled trial of Lichtenstein's repair with polypropylene mesh versus Surgisis Inguinal Hernia Matrix. Am J Surg. 2009;198:303-312. 